\title{
Construcción y validación de la Escala de compromiso hacia las tareas escolares en las clases para los estudiantes del nivel primario de educación
}

Construction and validation of the scale of engagement to school tasks in classes for the students of the primary level of education

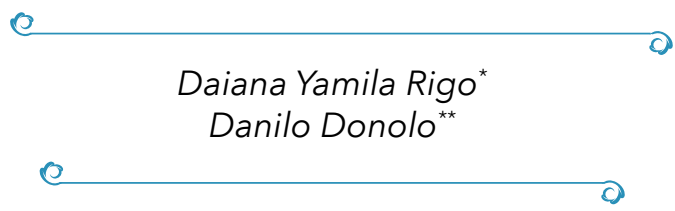

Recibido 02. 09. $2018 \bullet-$ Arbitrado 19.11. $2018 \bullet$ Aprobado 25.11. 2018

Licenciada en Psicopedagogía, Magíster en Piscología de la Educación y Doctora en Psicología. Docente de la UNRC e investigadora asistente del CONICET, Argentina, daianarigo@ hotmail.com

* Maestro Normal Nacional, Profesor y Licenciado en Ciencias de la Educación y Doctor en Ciencias de la Educación. Profesor consulto de la UNRC e investigador principal del CONICET, Argentina, donolo@gmail. com

\section{Resumen}

El presente escrito describe el proceso de construcción y validación de un instrumento para valorar el compromiso de los estudiantes hacia las tareas escolares en las clases del nivel primario de educación. La construcción de la escala contempla ítems incorporados por otros instrumentos en el marco de investigación sobre participación, interés y autorregulación en escuelas primarias, conforme a tres dimensiones, a saber: compromiso afectivo, conductual y cognitivo. Participaron del estudio 339 estudiantes de colegios primarios del Departamento de Río Cuarto, Córdoba, Argentina. Los resultados encontrados a partir del análisis factorial confirmatorio, muestran que los datos recabados se ajustan al modelo de tres factores. Asimismo, la escala cumple con unos niveles aceptables de fiabilidad. De esta manera, el instrumento construido y adaptado cuenta con características psicométricas adecuadas al contexto de estudio.

Palabras clave: compromiso, escuela, interés, autorregulación, evaluación, tarea escolar. 


\section{Abstract}

This paper presents the process of construction and validation of an instrument to assess the engagement of students to school tasks in the primary education classes. The construction of the scale includes items incorporated by other instruments in the framework of research on participation, interest and self-regulation in primary schools, according to three dimensions, namely: affective, behavioral and cognitive engagement. Participating in the study were 339 primary school students from the Department of Río Cuarto, Córdoba, Argentina. The results obtained from the confirmatory factor analysis show that the data collected are adjusted to the three factor model. They also meet acceptable levels of reliability. In this way, the instrument constructed and adapted has psychometric characteristics appropriate to the study context.

Keywords: engagement, school, interest, self-regulation, assessment, school tasks.

\section{Introducción}

El estudio del compromiso en contextos educativos tomó relevancia en el último tiempo, como la clave para trabajar problemáticas de bajo rendimiento, altos niveles de aburrimiento, fracaso y deserción (Fredricks y McColskey, 2012). No obstante, a pesar de su aparente utilidad, el compromiso de los estudiantes sigue siendo un concepto borroso con investigadores que contemplan definiciones ambiguas e inconsistentes con mediciones igualmente difusas.

Varios estudios recientes se han centrado en definir este meta-constructo, preparando el escenario para futuros estudios. Fundamentalmente, se observó en principio muchos trabajos orientados a marcar los límites conceptuales del tópico, crecimiento que no fue proporcional a las investigaciones empíricas, en parte por la falta de instrumentos para medir la complejidad de un concepto multidimensional (Reschly y Christenson, 2012; Cuevas, Sánchez-Oliva, Fernández-Bustos, 2016; Fredricks, Filsecker y Lawson, 2016). Al respecto, a finales de siglo XIX Newmann (1986) remarcaba la dificultad de definir operacionalmente el compromiso de los estudiantes, a pesar de evocarlo cuando lo vemos o de percibir su ausencia, de inmediato, en la clase.

Esta complejidad, menciona Lester (2013), se definió en términos generales desde los años 50 y 60, y tan sólo, en la actualidad se están produciendo investigaciones que ayudan a clarificar el significado y los alcances del compromiso. Las definiciones que más circulan entre las referencias de 
especialistas, son la de Newmann, Wehlage y Lamborn (1992) como la inversión psicológica y esfuerzo que los estudiantes dirigen hacia el aprendizaje y la comprensión del conocimiento que la tarea escolar intenta promover; y la de Fredricks y McColskey (2012), quienes entienden al compromiso en términos de participación, acción, o las manifestaciones conductuales, emocionales y cognitivas del individuo en interacción con el contexto. Lo que significa que la implicación de los estudiantes es maleable y sensible a las variaciones o modificaciones del contexto de la clase. Aunque la variedad de conceptualizaciones fueron resumidas en una revisión de Appleton, Christenson y Furlong (2008) a partir de la cual se extrajeron diversas dimensiones asociadas al compromiso, como participación en el trabajo escolar, compromiso académico, implicación del estudiante, participación del estudiante en o con la escuela, e identificación con la escuela. Asimismo, en los intentos de clarificar el constructo, Chistenson, Reschly y Wylie (2012) remarcan que algunos estudios comprenden al compromiso como un proceso, mientras que otros lo sitúan como un mero resultado.

Sobre la perspectiva del compromiso como meta-constructo, en las diferentes investigaciones, el involucramiento aparece asociado a dos o tres componentes. Los modelos que incluyen dos elementos, dejan afuera la dimensión cognitiva, mientras que, los de tres componentes que la incluyen (Appleton et al., 2008), parten de una conceptualización tripartita que encierra una dimensión conductual (participación, esfuerzo y conducta positiva); una afectiva (interés, identificación, sentido de pertenencia, actitud positiva hacia el aprendizaje); y una cognitiva (autorregulación, metas de aprendizaje) de la implicación escolar. Aunque los indicadores varían en función de querer atender el compromiso con la escuela o con la clase y las tareas que allí se desarrollan; a la actualidad muchos trabajos se han realizado para caracterizar los componentes que subyacen a cada dimensión. Específicamente, los trabajos orientados al compromiso con la clase, definen a la dimensión afectiva en términos de interés hacia las tareas escolares propuestas, al componente conductual lo delimitan en la participación de los estudiantes en las actividades de aprendizaje, y al cognitivo lo encuadran en los procesos y estrategias de autorregulación y aprendizaje profundo que despliegan los estudiantes para resolver una actividad de aprendizaje (Fredericks, Blumenfeld y Paris, 2004; Fredricks y McColskey, 2012; Shernoff et al., 2016).

En el presente estudio se retoman los aportes de Kong, Wong y Lam (2003) y Fin (1993) para conceptualizar los componentes. En caso del compromiso conductual se consideran los aspectos como: el proceso continuo de participación de los estudiantes, reflejado en la iniciativa de participar en las 
actividades de aprendizaje, mantener la atención hacia el docente y responder a consultas durante el desarrollo de la clase; en cuanto al compromiso afectivo, se tienen en cuenta el grado de interés, asociado al disfrute, que refleja la orientacion del sujeto hacia las tareas escolares, al igual como las reacciones negativas y positivas de los alumnos hacia los profesores, los compañeros de clase y las tareas académicas. En general, un buen indicador es el interés hacia el trabajo en el aula, siendo una menor implicación afectiva afín a conductas tales como el aburrimiento, la ansiedad, la frustración y los nervios en el aula (Eccles et al., 1983; Rigo y Donolo, 2014; Rigo, 2017).

El compromiso cognitivo, entendido como el nivel en el cual los alumnos están dedicando esfuerzos cognitivos hacia el aprendizaje, la comprensión, el dominio disciplinar y la adquisición de habilidades que el trabajo académico intenta promover. Un alumno comprometido a nivel cognitivo, es un estudiante estratégico, que autorregula los procesos de aprendizaje, en tanto hace uso de estrategias para planificar, monitorear y evaluar la realización de las tareas escolares (Sperling, Howard, Miller y Murphy 2002; Sperling, Ramsay, Reeves, Follmer y Richmond, 2016; Reeve, 2013; Rigo y Donolo, 2014; Rigo, 2017).

Con respecto al campo de medición del constructo una revisión coordinada por Fredricks et al. (2011) muestra la disponibilidad de instrumentos para medir el constructo en todos los niveles educativos. Clasifican las medidas según el grado en que reflejan la naturaleza multidimensional del término, el tipo de instrumento y el objeto del compromiso. De las 21 medidas que mencionan, sólo 6 valoran las tres dimensiones, afectiva, conductual y cognitiva. Con relación al formato y el objeto de medición, 14 son de auto-reporte, y 9 de estos están redactados para reflejar la participación general en la escuela, y los otros 5 indican el nivel de compromiso con la clase; se mencionan también 3 reportes que completan los docentes, de los cuales 2 pueden utilizarse para informar sobre la participación de los estudiantes en cualquier tema y el tercero para valorar la participación en el área de lectura; por último, de las grillas de observaciones, 2 proporcionan un sistema de codificación para la observación de comportamiento dentro y fuera de la tarea o la clase de un estudiante individual, y 2 evalúan el compromiso de aula de todos los estudiantes en la clase. Asimismo, respecto a la población para la cual fueron construidos, la mayoría se orienta al nivel medio y superior de educación, siendo que, para el nivel primario se encuentran menor cantidad de instrumentos, entre ellos no hay medidas de auto-informe para estudiantes, solo reportes de los docentes o grillas de observación que, únicamente, miden las dimensiones afectiva y conductual. 
De la revisión se desprende no sólo el acotado desarrollo de instrumentos para valorar el compromiso de los estudiantes que cursan el nivel primario de educación, sino, también, la ausencia de escalas que midan todas las dimensiones. Al respecto, Toll, Drefs y Lock (2016) exponen que el compromiso cognitivo presenta cierta dificultad de medición para los maestros, en gran parte porque es un proceso interno y no es fácilmente observable en el aula. En contraste, la dimensión conductual se manifiesta a partir de acciones observables y externas como la participación en las tareas de aprendizaje, y al igual que el compromiso afectivo a través del interés presentado ante las actividades propuestas. Cada componente es vital para una comprensión completa de la implicación de los estudiantes.

En el marco definido sobre la medición del compromiso, las medidas de auto-informe se presentan como la alternativa más promisoria para trazar un mapa del compromiso de los estudiantes, en sus tres dimensiones contempladas. Al respecto, Greene (2015) resalta la importancia de los instrumentos para examinar las percepciones de estudiantes en relación a su compromiso mientras estudian. Considerando un desarrollo escaso en torno al constructo en cuestión, el desafío de elaborar escalas que permitan medir la implicación hacia las tareas escolares emerge como necesidad a satisfacer, permitiendo aportar a futuras propuestas educativas orientadas a promover la implicación de los estudiantes (Hart, Stewart y Jimerson, 2011).

A continuación, detallamos las etapas del proceso de construcción y validación de la escala para medir compromiso en niños que cursan el nivel primario de educación, tomando como objeto de valoración las tareas de aprendizaje que tienen lugar en las clases. El desafío asumido, se une a la necesidad de contar, a futuro, con valoraciones que nos permitan estimar el impacto de los cambios en la configuración de las actividades escolares en el involucramiento de los estudiantes.

\section{Método}

\section{Muestra}

Se trabajó con un muestreo no probabilístico en el que participaron 339 niños que cursaban el segundo ciclo del nivel primario de educación en colegios del Departamento de Río Cuarto, Córdoba. De total de participantes, la edad mínima registrada fue de 9 y la máxima de 12 años $(\bar{X}=10,41 ; S=1,074)$. Respecto a la distribución por género, la mayoría eran varones (51\%) y en 
relación con el grado de escolaridad el $31 \%$ estaba en $4^{\text {to }}$, el $33 \%$ en $5^{\text {to }}$ y el resto en $6^{\text {to }}$.

Para acceder a la recolección de datos, se contó con la autorización de la inspección regional de educación primaria para acceder a las escuelas, se solicitó el consentimiento informado de los padres para la participación de los niños en el estudio, y se contemplaron las reglamentaciones éticos tanto de organismos nacionales como internacionales de investigación (CONICET, Comité de Ética de la Universidad Nacional de Río Cuarto y normas APA) sobre el resguardo del anonimato.

\section{Construcción de la escala. Procedimiento inicial}

Para desarrollar la escala de compromiso hacia las tareas escolares se tomó como referencia los pasos especificados por Zapata Rotundo y Canet Giner (2008) para la construcción de una escala de medición. En primer lugar, revisada la literatura y definidas las dimensiones que componen al compromiso, se pasó a formular un conjunto de ítems que constituirán el instrumento de valoración, realizando una revisión de las escalas elaboradas con anterioridad, que cubrieran los dos requisitos que definimos para su construcción, para niños escolarizados en el nivel primario de educación y compromiso hacia las actividades de aprendizaje $\mathrm{y}$, además, evaluaran las dimensiones conforme a la definición teórica adoptada.

Específicamente, tras la búsqueda realiza se seleccionaron dos instrumentos que cubrían los criterios expuestos, a saber: The Student Engagement in the Mathematics Classroom Scale de Kong, Wong y Lam (2003) y Metacognitive Awareness Inventory-versión A (Jr. MAI) de Sperling et al. (2002). Ambos, tienen la particularidad de cubrir la población de niños con edades entre los 9 y 12 años, lo que abarca en Argentina el segundo ciclo de la escolarización dentro del sistema formal de la escuela primaria, que comprende los grados

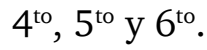

Asimismo, atendiendo a la definición de las dimensiones del primer instrumento de Kong, Wong y Lam (2003), se seleccionaron las siguientes sub-escalas: Interés, para evaluar compromiso afectivo; Atención-Participación, como indicador de compromiso conductual, ambas compuestas por 6 ítems; y Estrategias Profundas para valorar compromiso cognitivo, con 7 ítems, más otros 6 ítems que miden los procesos de autorregulación, integrados en esta última dimensión, del inventario de Sperling et al. (2002). En ambos casos, se solicitó la autorización de los autores para usar la escala y adaptar los ítems a nuestro contexto de investigación. 
Las sub-escalas utilizadas para el presente instrumento se adaptaron de acuerdo con el procedimiento habitual de traducción transcultural de escalas, el cual se realiza en tres etapas. En primer lugar, la escala original en inglés fue traducida al español: una persona bilingüe tradujo los originales del inglés al español, y otra persona tradujo estas versiones de nuevo al inglés. Luego, un grupo, formado por dos traductores y dos expertos en aprendizaje escolar y compromiso, seleccionaron los ítems que habían conservado el significado original. Seguidamente, se redactaron las instrucciones y el formato para la escala, eligiendo la técnica de escalamiento más utilizada de tipo Likert de 5 puntos, donde el valor $1=$ Siempre, $2=$ Casi siempre, $3=$ Normalmente, $4=\mathrm{A}$ veces y $5=$ Casi nunca (Zapata Rotundo y Canet Giner, 2008).

La escala inicial quedó conformada por un total por 25 ítems, versión que se aplicó a 50 alumnos de primaria que cursaban sexto grado para una evaluación inicial de la adecuación de los ítems, y se procedió a realizar las modificaciones pertinentes respecto al vocabulario apropiado al contexto de aplicación, cuidando la finalidad del instrumento orientado a medir el compromiso de los estudiantes hacia las tareas de aprendizaje. De ese primer análisis, se eliminaron algunos ítems referidos a la sub-escala estrategias profundas (cuando aprendo un nuevo tema, me pregunto si las cosas que he aprendido se pueden aplicar a la vida real; dedico tiempo extra-clase para profundizar la comprensión de aspectos interesantes de cada materia o actividad; intento relacionar lo que aprendí en la clase con la vida real o con otras materias; uso mi tiempo libre para estudiar los temas que se han discutido en clase) y de autorregulación (cuando termino la tarea, me pregunto si aprendí lo que deseaba aprender; presto mucho atención a la información importante; realizo esquemas o diagramas para ayudarme a comprender mientras estudio), donde se registraron correlaciones bajas $(<0,30)$ con el resto de los ítems que miden la dimensión cognitiva; a la vez, tras su eliminación se observó que el coeficiente de confiabilidad de la sub-escala aumentó. Cabe destacar, que antes de proceder a su eliminación, se revisaron nuevamente y se administró otra vez el instrumento, sin lograr mejorar el índice de correlación. Finalmente, la escala en su versión final quedó conformada por 18 ítems, como se presenta en la Tabla N 1, la versión para aplicar se presenta en el Apéndice 1, junto a las instrucciones generales. 
Tabla N 1: Escala de compromiso hacia las tareas escolares en las clases del nivel primario

\section{Ítem}

En la clase los contenidos y las actividades son interesantes y agradables.

Me gusta aprender y me interesan las materias y las actividades de aprendizaje.

Siento una sensación de satisfacción cuando realizo las tareas en clase.

Siempre tengo curiosidad por aprender cosas nuevas y me gusta hacer las actividades.

Me siento emocionado cuando empezamos un nuevo tema en clase.

Estoy muy interesado en estudiar nuevos temas, porque es placentero para mí.

Escucho con atención las explicaciones del maestro sobre la tarea a resolver.

En la discusión de temas nuevos participo activamente y defiendo mis opiniones.

Realmente me esfuerzo en clase y en las tareas escolares.

Me concentro mucho cuando la maestra introduce temas nuevos o tareas.

Utilizo distintas estrategias para comprender lo que la maestra enseña y hacer las actividades.

Siempre participo en el debate de la clase para resolver las tareas.

Cuando resuelvo las tareas, reflexiono sobre lo aprendido y trato de lograr una nueva comprensión de lo que sé

Cuando leo para hacer la tarea, trato de distinguir la información más importante en lugar de sólo leer el texto.

En las tareas de aprendizaje, siempre intento plantearme preguntas porque me ayudan a comprender aspectos centrales del tema.

Pienso en varias maneras de resolver una tarea y luego escojo la mejor opción.

Pienso sobre lo que tengo que aprender antes de empezar a trabajar.

Me pregunto qué tan bien estoy haciendo mientras aprendo algo nuevo.
AR4

\section{Dimensión *}

AF1

AF2

AF3

AF4

AF5

AF6

C1

C2

C3

C4

C5

C6

PS1

PS2

PS3

AR5

AR6

* $\mathrm{AF}=$ compromiso afectivo; $\mathrm{C}=$ compromiso conductual; $\mathrm{PS}$ y $\mathrm{AR}=$ compromiso cognitivo, estrategias profundas y procesos de autorregulación respectivamente.

Antes de proceder a realizar el análisis de fiabilidad y validez de la versión final -con el programa estadístico SPSS y AMOS versión 16, tras tomar una muestra más amplia, se identificó la presencia de datos extremos (outliers) a través de la distancia de Mahalanobis al cuadrado (D2), eliminando aquellos casos, cuya distancia estuviera asociada a una probabilidad cercana a 0,001 (González, Abad y Lèvy, 2006), quedando definitivamente un n=339. Luego, se procedió a calcular la normalidad multivariada a partir del coeficiente de curtosis multivariada de Mardia, cuyos valores críticos (c.r.) deben de caer dentro del intervalo [-1,96 - 1,96]; para los datos recolectados se comprueba una distribución normal multivariada (c.r. $=<1.96)$, habilitando el uso de 
método de Máxima Verosimilitud (MV) para estimar los valores de los parámetros desconocidos y sus respectivos errores de medición. Finalmente, se calculó la prueba de Kaiser-Meyer-Olkin (KMO) y la de esfericidad de Bartlett. Los resultados de ambas pruebas confirman la comunalidad entre los ítems dado que la medida de adecuación muestral $\mathrm{KMO}=0,919$ y la prueba de esfericidad de Batlett arrojó un p-valor p-valor $=0,000$, con $X^{2}=1667,609$ y $\mathrm{gl}=153$.

\section{Evaluación de la validez y fiabilidad de la escala}

Antes de llevar a cabo el análisis factorial confirmatorio, se realizó un análisis exploratorio con el objetivo de comprobar la estructura factorial de la nueva versión del instrumento respecto a los planteamientos teóricos. Los resultados, tal como se observan en el Tabla 2, muestran que estructura de tres factores explica un $47 \%$ de la varianza del constructo valorado, es decir, que el compromiso está conformado por tres dimensiones, afectiva, conductual y cognitiva.

Tabla N 2: Matriz de componentes principales

\begin{tabular}{cccc}
\hline Ítems & \multicolumn{3}{c}{ Componente } \\
& F1 & F2 & F3 \\
\hline EP1 &, 616 & & \\
EP2 &, 490 & & \\
EP3 &, 522 & & \\
ME4 &, 547 & & \\
ME5 &, 521 & & \\
ME6 &, 481 & & \\
IN1 & &, 628 & \\
IN2 & &, 662 & \\
IN3 & &, 556 & \\
IN4 & &, 649 & \\
IN5 & &, 560 & \\
IN6 & &, 657 & \\
PA1 & & &, 579 \\
PA2 & & &, 357 \\
PA3 & & &, 648 \\
PA4 & & &, 559 \\
PA5 & & & \\
PA6 & & & \\
\hline
\end{tabular}


Seguidamente, se pasó a confirmar la estructura factorial de tres dimensiones de la escala para valorar compromiso. En la Figura 1 se observa el path diagram para el modelo propuesto.

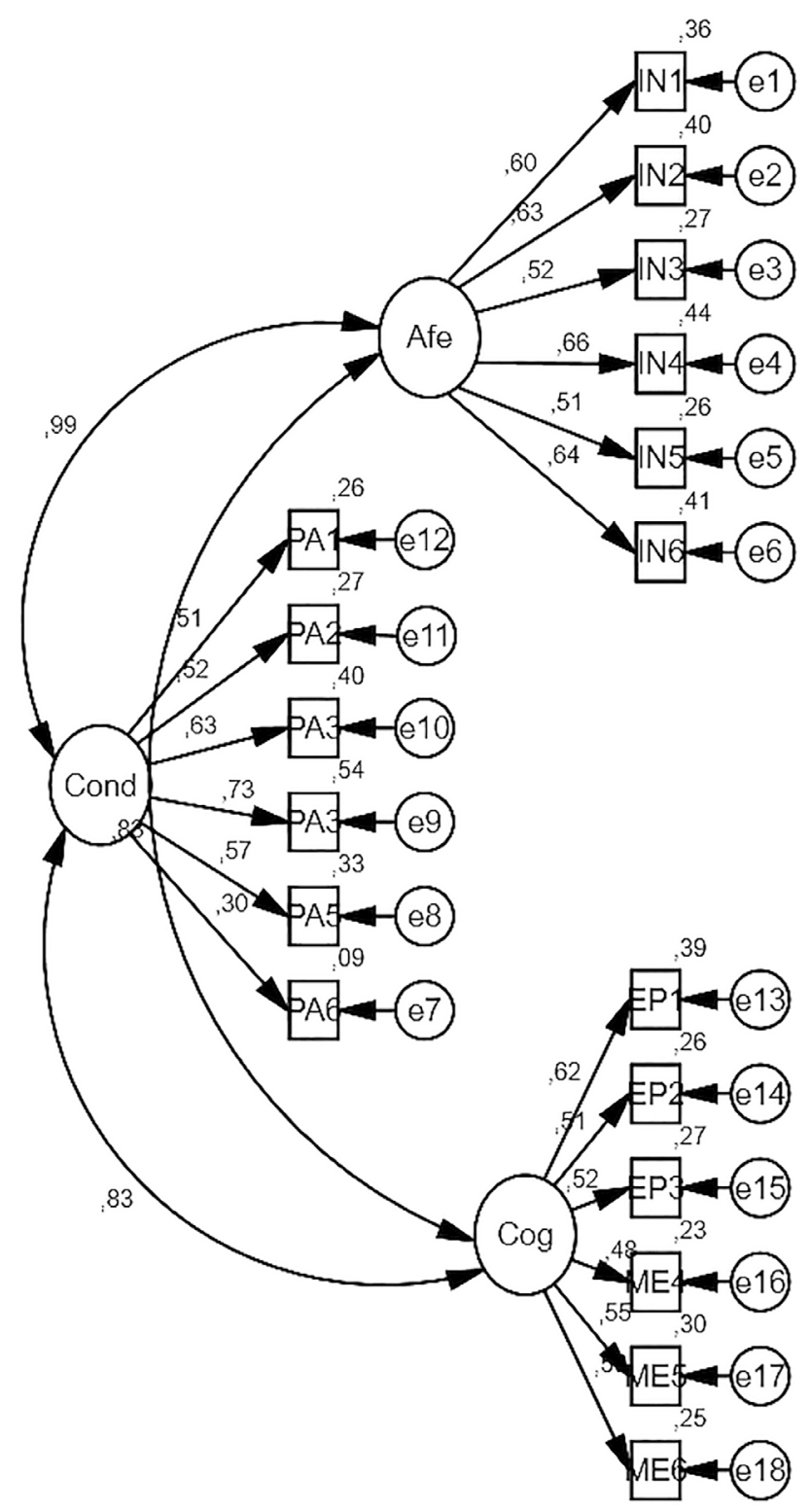

Figura $N$ 1: Diagrama de flujos para el Análisis Factorial Confirmatorio de la escala de compromiso, 3 factores 
Como indicador de bondad de ajuste utilizamos múltiples indicadores para evaluar la calidad del modelo. Entre los más destacados están la prueba de significación estadística de $X^{2}$, el cual reporta un buen ajuste cuando se acepta la hipótesis nula ( $\mathrm{p}>0,05)$; el GFI (Goodness of Fit Index) y el GFI (Comparative Fit Index), cuyos valores oscilan entre 0 y 1 , siendo aceptable valores cercanos o superiores a 0,90; y el RMSEA (Root Mean Square Residual), considerado óptimo cuando sus valores se encuentran entre 0,06 y 0,08, y del cual se recomienda informar el intervalo de confianza -IC- (Fernández Pulido, 2008; Orgaz Baz, 2008; Ruiz, Pardo y San Martín, 2010). Los resultados para cada índice se sintetizan en la Tabla 2.

Tabla N 3: Índices de bondad de ajuste para el modelo propuesto

\begin{tabular}{cccccc}
\hline$X^{2}$ & gl & p & GFI & CFI & RMSEA (IC al 90\% de confianza) \\
\hline 222,615 & 132 & 0,0001 & 0,929 & 0,938 & $0,046(0,036-0,056)$ \\
\hline
\end{tabular}

La bondad de ajuste del modelo indica que las tres dimensiones -afectiva, conductual y cognitiva- factorizan para el constructo compromiso, con índices que muestran valores dentro de los límites aceptables. No obstante, se observa que la prueba de significación estadística $X^{2}$ arroja un nivel de significación inferior a 0,05 , lo cual indica que la estructura teórica propuesta en el modelo es significativamente distinta a la indicada en la matriz de covarianza de los datos, en tanto es una prueba muy sensible al tamaño de la muestra. Por este motivo, otro criterio adoptado para evaluar el ajuste global del modelo es mirar no tanto el nivel de significación obtenido, sino la razón $X^{2} / \mathrm{gl}$, donde valores menores a 5 corresponderían a un ajuste razonable (Marsch y Hocevar, 1985). Claramente, el modelo propuesto satisface este último criterio.

También de la Figura 1, por un lado, es interesante resaltar que los pesos factoriales son igual o superiores a 0,30 mostrado la importancia que tiene el factor en la determinación de la dimensión valorada, al igual que lo muestran las correlaciones múltiples al cuadrado $\left(\mathrm{R}^{2}\right)$; por otro lado, se informa que la significación de todos los estimadores es igual a $\mathrm{p}<, 01$. Esos resultados, junto a la bondad de ajuste del modelo, muestran que el instrumento mide las tres dimensiones que configuran el compromiso de los estudiantes frente a las tareas de aprendizaje en la clase de nivel primario de educación.

Con respecto al análisis de fiabilidad de la escala, fue calculada por medio del alpha, superando el valor $a=0,70$ para cada ítem; mientras que, los valores para cada sub-escala fue igual a 0,712 para la dimensión afectiva; de 0,764 para la conductual; y de 0,705 para la cognitiva. Los valores sugieren una confiabilidad aceptable para el instrumento en general, ítem y sub-escalas. 
Para finalizar, la interpretación de los resultados de la escala validada, sin presentar ítems que deban revertirse, las puntuaciones bajas deben interpretarse como indicador de un mayor compromiso, según se dispone la escala Likert para el presente cuestionario. Específicamente, se recomienda contemplar tres puntos de corte, delimitados por los cuartiles -Q-: puntuaciones iguales o menores $Q_{1}$, por encima del $Q_{1}$ e iguales al $Q_{2}$, y por encima del $\mathrm{Q}_{2}$ e iguales o superiores al $\mathrm{Q}_{3}$. Teniendo como referencia los valores de la siguiente Tabla $\mathrm{N}$ 4:

Tabla $N$ 4. Pautas de interpretación. Puntuaciones de referencia para cada dimensión de compromiso

\begin{tabular}{ccccc}
\hline & & Cognitiva & Afectiva & Conductual \\
\hline \multicolumn{2}{c}{ Mínimo } & 6 & 6 & 6 \\
\multicolumn{2}{c}{ Máximo } & 27 & 29 & 30 \\
\hline \multirow{3}{*}{ Percentiles } & 25 & 12 & 10 & 11 \\
& 50 & 15 & 14 & 14 \\
& 75 & 18 & 17 & 17 \\
\hline
\end{tabular}

\section{Consideraciones finales}

El objetivo del presente estudio fue desarrollar una escala de medición del compromiso específica para estudios encaminados en el nivel primario de educación de Argentina, que tuvieron como objeto la valoración de la implicación hacia las tareas escolares que los estudiantes realizan y resuelven en las aulas, con el propósito final de contar con una medida con adecuadas características psicométricas que permita evaluar, a futuro, el impacto que nuevas configuraciones instructivas posibiliten sobre la promoción de la implicación de los estudiantes en la dimensión afectiva, conductual y cognitiva.

El desarrollo de instrumento ajustado a la población que interesa investigar es de crucial importancia, considerando que el compromiso con tareas es maleable y se modifica según las trasformaciones que tienen lugar en los contextos educativos, referidos a las actividades escolares que se desarrollan como andamio de nuevos aprendizajes. Es el aumento por el interés de profundizar sobre el compromiso del estudiante con adecuadas valoraciones, lo que cimienta las bases para pensarlo como variable que posibilita tomar decisiones basadas en datos situados (Reschly y Christenson, 2012).

De allí el valor de construir y validar instrumentos para objetivos y poblaciones específicas se sustenta en la ventaja de los auto-informes que evalúan 
mejor un constructo cuando están establecidas y definidas las bases teóricas de un estudio, su dominio y unidad de análisis. Como afirman Krueger y Kling (2000), las recomendaciones universales, incluso dentro de un área específica, son precipitadas porque diversos factores contextuales pueden influir en la validez y utilidad de los instrumentos.

La claridad conceptual y el rigor metodológico se consideran como requisito previo para avanzar en la construcción de intervenciones y programas para promover la participación, el interés y el aprendizaje autorregulado de los estudiantes. A partir del trabajo realizado se ha logrado construir y validar con una muestra de estudiantes de nivel primario de educación un instrumento de auto-informe con adecuadas propiedades psicométricas para medir el compromiso con las tareas escolares que tienen lugar dentro del aula. El análisis factorial exploratorio permitió identificar la existencia de un modelo de tres factores, el cual fue demostrado mediante el análisis factorial confirmatorio con adecuada confiabilidad de sus ítems y sub-escalas.

La estructura final indica que las tres dimensiones, afectiva, conductual y cognitiva constituyen tres aspectos diferentes, aunque vinculados al compromiso con la tarea escolar, conclusión consistente tanto con los fundamentos teóricos y los instrumentos revisados para la construcción de la presente escala.

Finalmente, como prospectiva de investigación se sugiere la utilización de otras pruebas a modo de tener una apreciación más real de cada dimensión del compromiso (Rigo, 2017). Greene (2015) advierte que las escalas de auto-evaluación tienen como debilidad ser más subjetivas, en tanto evalúan la percepción del sujeto sobre el compromiso que suponen tener, que está directamente relacionada con la capacidad introspectiva de cada individuo; en lugar de confiar en un solo instrumento, se necesita desarrollar otros enfoques de medición del compromiso con la tarea escolar, tales como grillas de observación, reportes de los docentes, o entrevistas en profundidad, generando modelos de investigaciones mixtas que permitan enriquecer los datos y valorar la influencia de las modificaciones en las tareas de aprendizaje sobre el compromiso de los estudiantes en próximas investigaciones.

\section{Referencias}

Appleton, J., Christenson, S., Furlong, M. (2008). Student engagement with school: critical conceptual and methodological issues of the construct. Psychology in the Schools, 45(5): 369-386. https://doi.org/10.1002/pits.20303 
Chistenson, S. Reschly, A., Wylie, C. (2012). Preface. En S. Chistenson, A. Reschly, \& C. Wylie (Edits.). Handbook Research on Student Engagement (pp. V-IX). New York: Springer.

Cuevas, R., Sánchez-Oliva, D., Fernández-Bustos, J. G. (2016). Adaptación y validación de la Escala de compromiso agéntico al contexto educativo español. Revista Mexicana de Psicología, 33(2), 135-142. https://www.redalyc.org/articulo. oa?id $=243056044005$

Eccles, J., Adler, T., Futterman, R., Goff, S., Kaczala, C., Meece, J., Midglay, C. (1983). Expectancies, values, and academic behaviors. En J. Spence (ed.) Achievement and achievement motives (pp. 75-146). San Francisco: W. H. Freeman.

Fernández Pulido, R. (2008). Modelos de medida y análisis factorial confirmatorio. En M., Verdugo, M. Crespo, M. Badía \& B. Arias (Coord). Metodología en la investigación sobre discapacidad. Introducción al uso de las ecuaciones estructurales, (pp. 29-41). Salamanca: Publicaciones del INICO.

Finn, J. (1993). School engagement \& student at risk. Washington, DC: National Center for Educational Statistics, Departmnet of Education. Recuperado http:// nces.ed.gov/pubs93/93470a.pdf

Fredricks, J., McColskey, W. (2012). The Measurement of Student Engagement: A Comparative Analysis of Various Methods and Student Self-report Instruments. En S. Chistenson, A. Reschly, \& C. Wylie (Edits.). Handbook Research on Student Engagement (pp. 763-782). New York: Springer.

Fredricks, J. A., Filsecker, M., Lawson, M. A. (2016). Student engagement, context, and adjustment: Addressing definitional, measurement, and methodological issues. Learning and Instruction, 43, 1-4. doi: 10.1016/j.learninstruc.2016.02.002

Fredricks, J., McColskey, W., Meli, J., Mordica, J., Montrosse, B., Mooney, K. (2011). Measuring student engagement in upper elementary through high school: a description of 21 instruments. Washington, DC: U.S. Department of Education, Institute of Education Sciences, National Center for Education Evaluation and Regional Assistance, Regional Educational Laboratory Southeast. Recuperado http://ies.ed.gov/ncee/edlabs

Fredriscks, J., Blumenfeld, O. \& Paris, A. (2004). School Engagement: Potential of the concept state of the evidence. Review of Educational Research, 74 (1): 59-109. https://doi.org/10.3102/00346543074001059

Gónzalez. N., Abad, J., Lèvy, J.P. (2006). Normalidad y otros supuestos en análisis de covarianzas. En J. Lévy. (Edit.): Modelización con estructuras de covarianzas (pp. 31-57). La Coruña: Netbiblo. 
Greene, B. A. (2015). Measuring Cognitive Engagement with Self-Report Scales: Reflections from over 20 years of research. Educational Psychologist, 50(1), 14-30. Recuperado http://dx.doi.org/10.1080/00461520.2014.989230

Hart, S., Stewart, K., Jimerson, S. (2011). The Student Engagement in Schools Questionnaire (SESQ) and the Teacher Engagement Report Form-New (TERF-N): Examining the Preliminary Evidence. Contemporary School Psychology, 15: 67-79. https://link.springer.com/article/10.1007/BF03340964

Kong, Q., Wong N., Lam, C. (2003). Student engagement in mathematics: Development of instrument and validation of construct. Mathematics Education Research Journal, 15 (1): 4-21. https://link.springer.com/article/10.1007/BF03217366

Krueger, R.F., Kling, K.C. (2000). Self-report. En A.E. Kazdin (Ed.). Encyclopedia of Psychology, 7 (pp. 220-224). New York: Oxford University Press

Lester, D. (2013). A Review of the Student Engagement Literature. Focus on Colleges, Universities and Schools, 7 (1). Recuperado http://www.nationalforum.com/ Electronic\%20Journal\%20Volumes/Lester,\%20Derek\%20A\%20Review\%20 of $\% 20$ the $\% 20$ Student $\% 20$ Engagement $\% 2$ Literature $\% 20$ FOCUS $\% 20$ V7 $\% 20$ N1\%202013.pdf

Marsch, H., Hocevar, D. (1985). Application of Confirmatory Factor Analysis to the Study of Self-Concept. First- and Higher Order Factor Models and Their Invariance Across Groups. Psychological Bulletin, 97(3), 562-582. http://dx.doi. org/10.1037/0033-2909.97.3.562

Newmann, F. M. (1986). Priorities for the future: Toward a common agenda. Social Education, 50(4): 240-250. https://eric.ed.gov/?id=EJ335096

Newmann, F., Wehlage, G., Lamborn, S. (1992). The significance and sources of student engagement. En D. M., Newmann (ed.). Student engagement and achievement in american secondary schools (pp. 11-39). New York: Teachers College Press.

Orgaz Baz, B. (2008). Introducción a la metodología SEM: conceptos y propósitos fundamentales. En M., Verdugo, M. Crespo, M. Badía \& B. Arias (Coord). Metodología en la investigación sobre discapacidad. Introducción al uso de las ecuaciones estructurales, (pp. 14-28). Salamanca: Publicaciones del INICO.

Reeve, J. (2013). How Students Create Motivationally Supportive Learning Environments for Themselves: The Concept of Agentic Engagement. Journal of Educational Psychology, 105 (3): 579-595. http://dx.doi.org/10.1037/a0032690

Reschly, A., Christenson, S. (2012). Jingle, Jangle, and Conceptual Hazines: Evolutions and Future Direction of the Egaggement Construct. En S. Chistenson, 
A. Reschly, \& C. Wylie (Edits.). Handbook Research on Student Engagement (pp. 3-19). New York: Springer.

Rigo, D. (2017). Docentes, tareas y alumnos en la definición del compromiso: investigando el aula de nivel primario de educación. Educação em Revista, 33: 1-24. Recuperado http://dx.doi.org/10.1590/0102-4698154275

Rigo, D., Donolo, D. (2014). Entre pupitres y pizarrones. Retos en educación primaria: el aprendizaje con compromiso. Educatio Siglo XXI, 32 (2): 59-80 59. Recuperado http://dx.doi.org/10.6018/j/202161

Ruiz, M., Pardo, A., San Martín, R. (2010). Modelos de educaciones estructurales. Papeles del Psicólogo, 31(1), 34-45.

Shernoff, D., Kelly, S., Tonks. S., Anderson, B., Cavanagh, R., Sinha, S., Abdi, B. (2016). Student engagement as a function of environmental complexity in high school classrooms. Learning and Instruction, 43: 52-60. Recuperado https://doi. org/10.1016/j.learninstruc.2015.12.003

Sperling, A., Ramsay, C., Reeves, P., Follmer, D., Richmond, A. (2016). Supporting students' knowledge construction and self-regulation through the use of elaborative processing strategies. Middle School Journal, 47 (3): 25-32. Recuperado http:// dx.doi.org/10.1080/00940771.2015.1135099

Sperling, R., Howard, B., Miller L., Murphy, C. (2002). Measures of Children's Knowledge and Regulation of Cognition. Contemporary Educational Psychology, 27: 51-79. doi:10.1006/ceps.2001.1091

Toll, K., Drefs, M., Lock, J. (2016). Measuring cognitive engagement. The Canadian Journal for Teacher Research. Recuperado https://www.teacherresearch.ca/ search?text $=$ toll

Zapata Rotundo, G., Canet Giner, T. (2008). Propuesta metodológica para la construcción de escalas de medición a partir de una aplicación empírica. Revista Electrónica Actualidades Investigativas en Educación, 8 (2): 1-26. Recuperado http://www.redalyc.org/articulo.oa?id=44713044004 


\title{
Apéndice 1.
}

\section{ESCALA DE COMPROMISO HACIA LAS TAREAS ESCOLARES}

\author{
Versión A - Nivel Primario de Educación \\ Para estudiantes
}

Rigo y Donolo, 2018

Nos interesa conocer cómo llevas a cabo las tareas escolares, qué haces cuando estudias y resuelves resolvés las tareas, qué interés en ellas tenés y cuál es tú participación hacia las actividades de aprendizaje desarrolladas en el aula.

NO hay respuestas correctas o incorrectas. Por favor, responde libre y espontáneamente con la mayor sinceridad posible.

Para responder, lee cada oración y toma en cuenta la escala de cinco puntos para dar una respuesta:

$1=$ Siempre

$2=$ Casi siempre

$3=$ Normalmente

$$
4=\mathrm{A} \text { veces }
$$

$$
5=\text { Casi nunca }
$$

Encierra con un círculo el número que se corresponda con la respuesta que elegiste. Por ejemplo:

Si para la primera oración tú respuesta es casi siempre, encierra con un círculo el 2

\begin{tabular}{|c|c|c|c|c|c|c|c|}
\hline AF 1 & $\begin{array}{l}\text { En la clase los contenidos y las actividades son interesantes y } \\
\text { agradables. }\end{array}$ & 1 & 2 & 3 & 4 & 5 & 1. \\
\hline C1 & $\begin{array}{l}\text { Escucho con atención las explicaciones del maestro sobre la } \\
\text { tarea a resolver. }\end{array}$ & 1 & 2 & 3 & 4 & 5 & 2. \\
\hline PS1 & $\begin{array}{l}\text { Cuando resuelvo las tareas, reflexiono sobre lo aprendido y trato } \\
\text { de lograr una nueva comprensión de lo que sé }\end{array}$ & 1 & 2 & 3 & 4 & 5 & 3. \\
\hline AF2 & $\begin{array}{l}\text { Me gusta aprender y me interesan las materias y las actividades } \\
\text { de aprendizaje. }\end{array}$ & 1 & 2 & 0 & 4 & 5 & 4. \\
\hline
\end{tabular}

\begin{tabular}{|l|l|l|l|}
\hline En la clase los contenidos y las actividades son interesantes y agradables & 1 & 2 & 3 \\
\hline
\end{tabular} 


\begin{tabular}{|c|c|c|c|c|c|c|c|}
\hline C2 & $\begin{array}{l}\text { En la discusión de temas nuevos participo activamente y } \\
\text { defiendo mis opiniones. }\end{array}$ & 1 & 2 & 3 & 4 & 5 & 5. \\
\hline PS2 & $\begin{array}{l}\text { Cuando leo para hacer la tarea, trato de distinguir la } \\
\text { información más importante en lugar de sólo leer el texto. }\end{array}$ & 1 & 2 & 3 & 4 & 5 & 6. \\
\hline AF3 & $\begin{array}{l}\text { Siento una sensación de satisfacción cuando realizo las tareas en } \\
\text { clase. }\end{array}$ & 1 & 2 & 3 & 4 & 5 & 7. \\
\hline C3 & Realmente me esfuerzo en clase y en las tareas escolares. & 1 & 2 & 3 & 4 & 5 & 8. \\
\hline PS3 & $\begin{array}{l}\text { En las tareas de aprendizaje, siempre intento plantearme } \\
\text { preguntas porque me ayudan a comprender aspectos centrales } \\
\text { del tema. }\end{array}$ & 1 & 2 & 3 & 4 & 5 & 9. \\
\hline AF4 & $\begin{array}{l}\text { go curiosidad por aprender cosas nuevas y me gusta } \\
\text { ividades. }\end{array}$ & 1 & 2 & 3 & 4 & 5 & 10. \\
\hline C4 & $\begin{array}{l}\text { Me concentro mucho cuando la maestra introduce temas nuevos } \\
\text { o tareas. }\end{array}$ & 1 & 2 & 3 & 4 & 5 & 11. \\
\hline AR4 & $\begin{array}{l}\text { en varias maneras de resolver una tarea y lu } \\
\text { pción. }\end{array}$ & 1 & 2 & 3 & 4 & 5 & 12. \\
\hline AF5 & $\begin{array}{l}\text { Me siento emocionado cuando empezamos un nuevo tema en } \\
\text { clase. }\end{array}$ & 1 & 2 & 3 & 4 & 5 & 13. \\
\hline C5 & $\begin{array}{l}\text { Utilizo distintas estrategias para comprender lo que la maestra } \\
\text { enseña y hacer las actividades. }\end{array}$ & 1 & 2 & 3 & 4 & 5 & 14. \\
\hline AR5 & $\begin{array}{l}\text { Pienso sobre lo que tengo que aprender antes de empezar a } \\
\text { trabajar. }\end{array}$ & 1 & 2 & 3 & 4 & 5 & 15. \\
\hline AF6 & $\begin{array}{l}\text { Estoy muy interesado en estudiar nuevos temas, porque es } \\
\text { placentero para mí. }\end{array}$ & 1 & 2 & 3 & 4 & 5 & 16. \\
\hline C6 & $\begin{array}{l}\text { Siempre participo en el debate de la clase para resolver las } \\
\text { tareas. }\end{array}$ & 1 & 2 & 3 & 4 & 5 & 17. \\
\hline AR6 & $\begin{array}{l}\text { Me pregunto qué tan bien estoy haciendo mientras aprendo algo } \\
\text { nuevo. }\end{array}$ & 1 & 2 & 3 & 4 & 5 & 18. \\
\hline
\end{tabular}

